\title{
NANOCARRIERS IN ORAL DELIVERY OF MACROMOLECULES: UPDATED INSIGHT
}

\author{
Saikh Mahammed Athar Alli
}

\author{
Assistant Professor, Department of Pharmaceutical Sciences, Faculty of Health Sciences, Shiats (Deemed to be
} University), Allahabad-221 007, Uttar Pradesh, India

Author's Email ID: atharodi@gmail.com, Phone and Fax No.: +91 5322684781

\begin{abstract}
Having handy reference asa source of outstanding knowledge for scientist engaged in developing nanocarrier based oral synergising their effect by amalgamating nanotechnology and presenting them as oral delivery system. Diverse methods and technology devised to achieve goal. All of them abreast increasing efficacy, specificity, tolerability and therapeutic index, and minimising toxicities. Each and every system has its own limitation and superiority over others. Amongst them nanocarrier based systems receiving attentions. These systems provide improved performance, stability, and patient compliance, and have potential for improved marketability. Their design and development progressed to make best formula through method of optimisation. This considered being a state of art involves multidisciplinary activity and creates difficulties as several causes needed to be achievable, associated with many features. Available literatures are unable to provide insight on issues relating their development while some are unhandy. In this regard, information collected from databases and presented as a handy note. This insight features delivery system of macromolecules (NBODSM). Macromolecule based biopharmaceuticals(proteins, peptides, monoclonal antibodies, and vaccines)explored for delivering associated proclaimed benefits, in diverse domain. Interest evoke for on issues relating development of NBODSM and hasapplicability inproductevolution. Presented information will be a helping hand and will offer an outstanding knowledge to developers, while designing them with excellent feature.

Key Words: Delivery, macromolecules, nanocarriers, nanotechnology, oral, strategy.
\end{abstract}

\section{INTRODUCTION}

Nanotechnology possesses enormous potential to produce products, with novel properties, in various domains, whose importance is expected to get enhanced, in near future. Novel materials and devices resulting from said technology have broad introduction potentiality in all domain including biotechnology, food, and pharmaceutical sector. In pharmaceutical field, introduction of nanotechnology is achieving in devising nanotherapeuticsand nanodevices. Semi-biological nanodevicesare multifunctionaloffering versatile therapeutic services and demonstrating unitary biochemical activities. While amalgamation of imaging and therapeutic function in a single nanodevice can provide prognostic information concurrent with therapeutic intervention $^{1}$.

Presently, pharmaceuticals containing nanotherapeuticsand nanodevicesare under extensive study for improving performance ${ }^{1}$. Ongoing attempt is to present them as disinfectants; biosensor or bio-tracer based diagnostic agent for detecting toxins, pathogens, volatile compounds, and organic components of body fluids; and for monitoring diseases ${ }^{1}$. Besides these they will revolutionise offering of device for site-specific controlled delivery of drug or drug targeting, and presenting of differential device-activity in dissimilar physiological environments, under direction of an external operator / physician $^{2-6}$.

Several nano sized pharmaceuticals were designed for oral delivery of nanotherapeuticmacromolecules but polymeric nanocarriers gaining popularity ${ }^{7}$. They allows paracellular and transcellulartranscytosis of macromolecules and site specific delivery or targeting, and provide desirable biopharmaceutical and pharmacokinetic profile ${ }^{8-11}$. Their consensus on increasing efficacy, specificity, tolerability and therapeutic index and prognostic on minimising toxicities are available ${ }^{3,4,12-17}$.

Polymeric nanocarriers with diverse functionality were designed using several materials and following various process or technique. They be either a vesicular system (i.e., nanovesicles, encapsulates drug in a cavity of polymeric membrane) or a matrix system (i.e., nanoparticles (NPs), in which drug is physically and uniformly dispersed $)^{7,12,18,19}$. All of these systems have limitations and superiority over others. Choice of system afoot on desired physicochemical properties and targeted therapeutic objective ${ }^{3,6,7}$. Biodegradable polymers of natural or synthetic origin used to wider extentwhile nonbiodegradable polymers are in rare use ${ }^{1,20}$. Table 1 depicts glimpse on IUPAC name and structure of polymers used for developing nanocarriers, while Table 2 presents glimpse on specialism of nanocarriers.

Adsorbing or grafting of molecules on surface of nanocarriers modifies its surface property that in turn modifies interaction with intestinal mucosa. Ligand molecule like antibodies, glycoproteins or peptides confers targeting ${ }^{9,22-25}$ while that of hydrophilic one,polyethylene glycol (PEG), improves transcytosis ${ }^{21}$.Adsorbing or coating of them with mucoadhesives (viz. chitosan) imparts mucoadhesive property ${ }^{1,5}$.

Present workinsights design andmanufacturing of NBODSMand underlinebalancing their physicochemical properties. Presented information has applicability in product evolution and will be a helping hand for developers, while designing them with excellent feature. 
Table 1: Depicts IUPAC name and structure of polymers used for developing nanocarriers

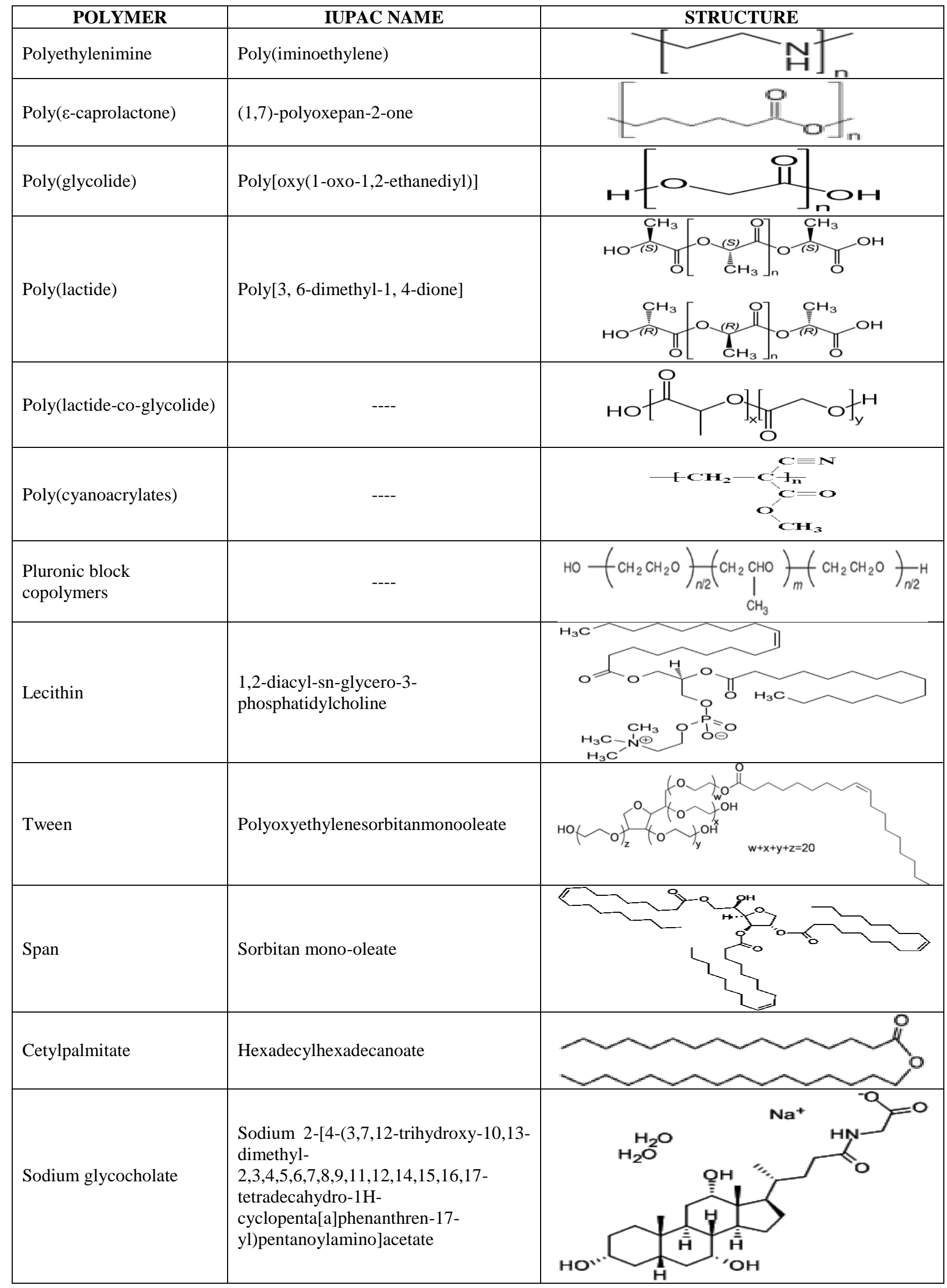




\section{NANOPARTICLES}

Physicochemical properties of NPs modulated with change in polymers and processing conditions. Modulation in hydrophobicity or surface charges, extent of drug loading, drug release profile, and biological behaviour make them versatility thereby amenable in orally delivering wide variety of drug with or without target specificity $5,16,26$.
Diverse methods followed for preparing them, depending on nature of polymer and drug. Use of process involving electrostatic interaction wise option, employ polyelectrolyte complexes and eliminates application of detrimental or aggressive in-process conditions ${ }^{27,28}$. While method requiring heat, organic solvent, sonication or vigorous agitation is unsuitable for biopharmaceuticals 1 , 20

\begin{tabular}{|l|l|l|l|}
\hline \multicolumn{3}{|c|}{ Table 2: Nanocarriers exploited for developing oral delivery system of macromolecules } \\
\hline $\begin{array}{c}\text { Type of } \\
\text { Nanocarriers }\end{array}$ & \multicolumn{1}{|c|}{ Composition } & Size Range & \multicolumn{1}{|c|}{ Specialism } \\
\hline Nanoparticles & $\begin{array}{l}\text { Natural or synthetic, biodegradable or non-biodegradable } \\
\text { polymers }\end{array}$ & $1-100 \mathrm{~nm}$ & $\begin{array}{l}\text { High kinetic stability, } \\
\text { static structure }\end{array}$ \\
\hline Liposomes & Natural or synthetic phospholipids & $20 \mathrm{~nm}-10 \mu \mathrm{m}$ & $\begin{array}{l}\text { Have } \\
\text { structure and are } \begin{array}{c}\text { ande } \\
\text { aiocompatible, safe, } \\
\text { and well-tolerated. }\end{array}\end{array}$ \\
\hline $\begin{array}{l}\text { Solid-lipid } \\
\text { nanoparticles }\end{array}$ & High melting point natural or synthetic fats & $50-1000 \mathrm{~nm}$ & \\
\hline Micelles & Ionic or non-ionic surfactants & $2-20 \mathrm{~nm}$ & \\
\hline Lipospheres & High melting lipid, phospholipids & $1-2-100 \mathrm{~mm}$ & \\
\hline $\begin{array}{l}\text { Submicron lipid } \\
\text { emulsions }\end{array}$ & Lipids, hydrophilic liquid, surfactants & \\
\hline
\end{tabular}

Natural, biocompatible polymers are alginate, agar, chitosan and its derivatives, dextran, gelatine, and so on. Synthetic, biocompatible and/or biodegradable are poly(cyanoacrylates) (PCA), poly(glycolide) (PGA), poly(lactide) (PLA), poly(lactide-co-glycolide) (PLGA), polyethylenimine or poly( $\varepsilon$-caprolactone) (PCL) $4-7,20,29$,

${ }^{30}$.Chitosan and its derivatives used popularly linked with biocompatible, non-toxic, mucoadhesive, targeting, and tight junctions (TJs) opening property ${ }^{29-33}$. Its limited solubility at $\mathrm{pH}>6.5$ being overcome by introducing alkyl groups to amine groups ${ }^{34}$. Polyelectrolyte complexes of 200-400 nm size formed with these ${ }^{27}$. PLA, PLGA, PCL biodegrade through hydrolysis while PCA with esterase. PCL degrade slowly than PLGA, thus suitable for extending delivery ${ }^{20,35}$. Chitosan used to greater extent while dextran, gelatine and alginate used to lesser extent ${ }^{1}$.

\section{NANOVESICLES}

Surfactants and amphiphilic polymers / copolymers form colloidal dispersions of molecular aggregates or vesicles ${ }^{13}$, 19, 36. Lipid-based vesicles are solid-lipid nanocarriers (SLN), micelles, lipid microspheres (lipospheres) and

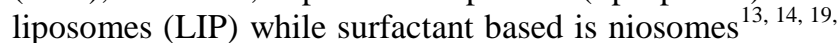
37, 38 . In contrast to NPs, which display a static and stable structure, they form a dynamic structure. These can carry drug in core or on corona and can solubilise poorly soluble drugs and partly protect drug from aqueous environment, thus preferred for poorly soluble drugs ${ }^{15}, 18,19$.SLN and niosomes are more stable and advantageous comparing LIP, can incorporate hydrophilic and/or hydrophobic drug. They protect incorporated drug from degradation; are biocompatible, safe, and well-tolerated; offer possibility

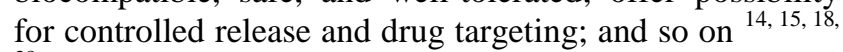
38. SLN exhibit beneficial solid state behaviour (crystallinity, polymorphism and thermal behaviour) as consequences of non-spherical particle shape, usually. Crystalline natured lipid core of which contribute additional beneficent features, including altering pharmacokinetics profiles, comparing others ${ }^{12,37}$.

Copolymer of PLA-PEG, PCL-PEG, or low molecular weight polyester-PEG is cheaper and safe but their use limited by involvement of sophisticated technology and machine, and organic solvents. Lecithin, tween 80, poloxamer 188 , span 85 , cetylpalmitate, and sodium glycocholate are safe ${ }^{38}$.Amphiphilic copolymers provide enhanced kinetic and thermodynamic stability comparing surfactants $^{14,15,18}$. Copolymer of pluronic-polyacrylic acid and poloxamer results pH-sensitive micelles ${ }^{39,} 40$. Derivatives of PEGylated $\alpha$-tocopherol and tocopherol polyethylene glycol succinate (TPGS), is biocompatible and suitable for delivering both soluble and poorly water soluble drugs ${ }^{14,15,19,38}$.Poloxamer solubilise drugs and enhance drug transport across the intestinal barriers ${ }^{11,41}$.

SLN formulated by solvent evaporation method or melt dispersion method but latter is advantageous as eliminates use of organic solvents ${ }^{42-44}$.LIP, lipospheres and niosomes are formed either by simple direct dissolution in water or by dissolving drug and polymer in organic solvents before solvent evaporation or dialysis $19,36,45,46$.

The polymers or copolymers use for micelles should (i) spontaneously self-assemble in water, (ii) enhance drug solubility by several fold (iii) provide high loading efficiency (iv) remain stable in the gastrointestinal (GI) tract, (v) be biocompatible and non toxic and (vi) easy to synthesise in commercial scale (vii) cheap and easily assessable.

\section{STABILITY OF NANOCARRIERS IN GI TRACT}


After oral administration, nanocarriers degrade with variation of $\mathrm{pH}$ levels and presence of enzymes or bile salts. Their composition strongly influences stability in GI tract. Nanocarriers prepared with insoluble polymers neither immediately degrades nor rapidly releases drug. While that with water-soluble polymers, forming polyelectrolyte one, are more likely to destabilise by $\mathrm{pH}$ or ionic strength. Kinetic stability of NPs is better than nanovesicles ${ }^{7,13}$.

\section{BIODISTRIBUTION OF NANOCARRIERS}

ATP-binding cassette (ABC) transporters (like Pglycoprotein (P-gp) and multi-drug resistance-associated proteins expressed by intestinal epithelial membrane) and various solute transporters facilitate absorption of nanocarriers and or drugs. The ABC transporters are ATP dependent thus limits absorption in a dose-dependent, inhibitable and saturable manner and can pump against steep of concentration ${ }^{41,47-50}$.

Upon crossing mucus the nanocarriers are traversing intestinal epithelium via paracellular pathway and/or transcytosis. Receptor-mediated transcytosis (internalization through endocytic pathways) is specific to intracellular locations and processes and mediated by enterocytes or $\mathrm{M}$ cells ${ }^{50}$. Endocytosis mechanism involves phagocytosis and pinocytosis. Phagocytosis is restricted to $\mathrm{M}$ cells and phagocytic immune cells ${ }^{47,51}$. Pinocytosis occurs by macropinocytosis (a transient process) and micropinocytosis (a constitutive pathway) ${ }^{5,41}$.

Micropinocytosis involves clathrin-mediated endocytosis (ClME), caveolae-mediated endocytosis (CaME) and clathrin- and caveolae-independent endocytosis. CIME involves either specific receptor-ligand interaction or nonspecific endocytosis. Macropinocytosis and CIME involves fusion with endolysosomes whereas CaME involve direct exocytosis by escaping endolysosomes. CaME involves formation of flask-shaped invaginations, a static structures with a size of 50 to $100 \mathrm{~nm}$, of the plasma membrane in cholesterol and sphingo lipid-rich microdomains ${ }^{47,50,51}$.

After absorption, drug and/or drug loaded nanocarriers can be included in cytoplasmic vesicles or diffuse in cytoplasm and be discharged in serosal spaces for gaining access to mesentheric lymph or blood ${ }^{5,26,41,48}$.The size, composition, surface characteristics and architecture of them along with physicochemical properties of polymer (viz. molecular weight, conformation, hydrophobicity, and so on) will monitor their absorption $9,10,47$. Water-insoluble polymers forms stable nanocarriers and are more likely absorbed as particles 5. Polymers forming less stable particles, polyelectrolyte complexes, vesicles or micelles will partly get dissociate and will not completely get absorbed as particle ${ }^{10,14,15,38}$. Nanocarriers taken up by absorptive enterocytes mainly delivered in blood. While those taken up by $M$ cells will transcytosis close to immune cells and are likely be delivered to the gut-associated lymphoid tissue and lymphoid cells ${ }^{5,9,10}$. Drugs encapsulated in NPs are not likely to be substrate of efflux pump while hydrophobic drugs forming core of micelles are more likely to be transported by efflux pumps ${ }^{5,10,11}$.Delivery mechanism of macromolecule through oral route is presented with Figure-1.

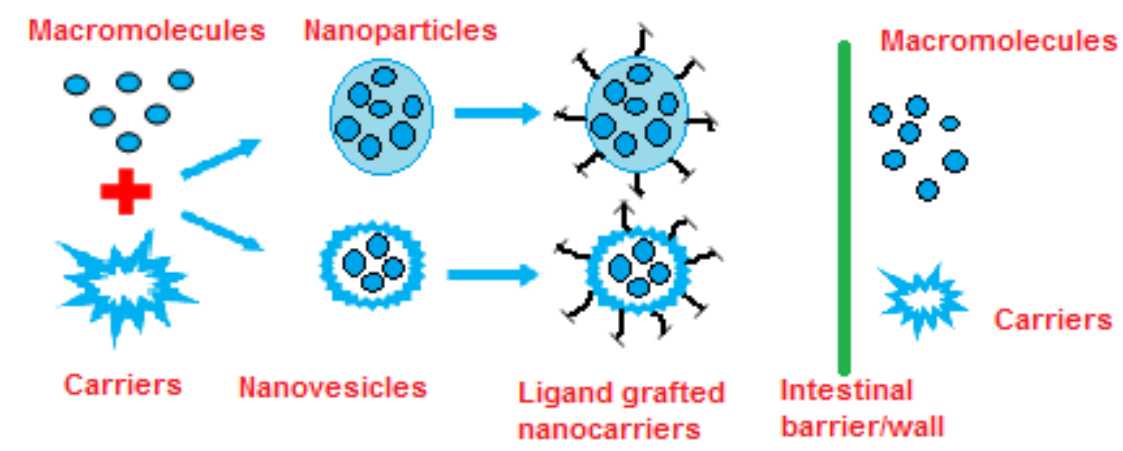

\section{Figure-1: Pictorial representation of delivery mechanism of macromolecules, via oral route}

\section{BIOAVAILABILITY TECHNIQUES}

IMPROVEMENT

In paracellular route junction-proteins present at tight or adherens junction restrains passage of macromolecules or aggregates with size more than one $\mathrm{nm}$. Consensus on nanocarriers is generally they do not transverse intestinal barrier by paracellular route $22,26,48$. While in transcellular route major limiting factor is intestinal mucosa which requires redresses for improving bioavailability of orally delivered macromolecules $5,8,16,22,41,47,50,52$.
Improvement in delivery of drug, by paracellular way, can be achievable by grafting modulators of junctional proteins on the surface of nanocarriers ${ }^{5,22}$. These modulators act directly or indirectly on TJ components, proteins or surface receptor resulting reversible opening of membranous barriers 32,33 . Some modulators disrupt the TJs via activation of protein kinase $\mathrm{C}$ and the adherens junctions by chelating calcium $22,47,50$.

Transcellular translocation of nanocarriers seems influencing by several physicochemical parameters including surface hydrophobicity, polymer nature and 
particle size. These factors influence their fate within enterocytes or $M$ cells while efflux pumps may strongly reduce extent of net drug uptake ${ }^{8,16,41,47,50}$.

Bioavailability or performance of nanocarriers ought to be improving through protecting drug from detrimental GI tract environment, prolonging their GI-residence time by mucoadhesion, their endocytosis, and permeabilising effect of polymer ${ }^{1,5,8,39,47}$.

\section{ENDOCYTOSIS}

Internalization of nanocarriers through endocytosis pathway depends on physicochemical characteristics and size of the particle and the cell type. Their transport can be enhanced by specifically targeting receptors (mediating endocytosis) through grafting or coating them with ligand, having affinity for receptors $8,22,41,47,49,50$.Several nonexhaustive receptors are there whose targeting can be done to increase their transport mediated by intestinal cells, globet cells or M cells 9, 24, 47, 49, 51, 53,54.Pattern recognition receptors (PRRs) such as toll-like receptor-4,plateletactivating factor receptor and $\alpha 5 \beta 1$ integrinpresent on the surface of $M$ cells considered as important in antigen transcytosis ${ }^{55}$. A few specific ligands of human M cells have been identified ${ }^{3,9,23}$. Grafting of these $M$ cell homing peptides (CKSTHPLSC (CKS9), CSKSSDYQC (CSC)) results in targeting of $\mathrm{M}$ cells and enhances transport ${ }^{23,56}$, ${ }^{57}$. Nanocarriers mimicking structure of pathogen or having affinity for $M$ cells is suitable for oral immunisation ${ }^{55}$. Integrins can be targeted with Arg-Gly-Asp (RGD) and aspartic-acid-based ligands. Among these RGD peptides cyclic peptides $\mathrm{c}(\mathrm{RGDfK})$ and $\mathrm{c}(\mathrm{RGDyK}), \mathrm{RGD} 4 \mathrm{C}$, and RGD10 showed high binding affinity and selectivity for integrin $^{54}$.

\section{TARGETING}

Many emerging nanocarriers are essentially delivery devices strongly emphasising on control of therapeutic action in time and space ${ }^{1}$.Targeted delivery devices depending on biologic affinity interactions (using vascular address system) and physical/chemical properties (enhanced permeability and retention effect), and pro-drug strategies (devices that are active only at preselected sites of disease) have emerged recent years 3, 6, 9, 16. Other related modalities are controlling the assembly or activity of devices at particular sites or at particular times in response to a signal provided by an external operator. Such signals are physical (a magnetic field) or biochemical (an enzyme transforming therapeutic to an active form) in nature $^{32,49}$. Up-regulating expression of some receptors using an appropriate stimulant is another strategy of targeting. Stimulation of these receptors with ligands, lipopolysaccharide or cytokines increases particle uptake by human follicle-associated epithelium cells ${ }^{5,8,9,56,57}$.

Some lectins can result targeting of adhesion molecules present on enterocytes and $\mathrm{M}$ cell membranes. Grafting of ligand is particularly attractive for oral vaccine delivery 9 , 23,57 . Adhesive factors (flagellin and invasins) derived from microorganisms, vitamins, and carbohydrates are used as ligands $1,5,58,59,60$.

Conjugating lectins to nanocarriers increases transport across the intestinal mucosa while grafting them with vitamin $\mathrm{B}_{12}$ allows targeting to intrinsic factor specific receptor ${ }^{10,50,60,61}$. Targeting of NPs and internalisation of micelles takes place via ClME. Endocytic translocation of small particles (with size 50-100 nm) mediated by enterocytes whilst larger particles mediated by $\mathrm{M}$ cells ${ }^{10}$, $41,47,50,51$.Chitosan and PLGA nanocarriers enhance transport via $\mathrm{ClME}^{31}$. Nanocarriers coated or grafted with ligands like folic acid, albumin and cholesterol, gets internalized by $\mathrm{CaME}^{51}$. Targeting of globet cell ${ }^{53}$, peyer's patches (PPs) ${ }^{45,56}$, dendritic cells (DCs) of PPs ${ }^{62}$ done by grafting ligands, having homing property or affinity forthem. Pluronic 85 unimers enter epithelial cells through caveolae-dependent and caveolae-independent pathways ${ }^{63}$. Thiamine-coated NPs captured by PPs ${ }^{58,56}$.

\section{MUCOADHESION}

Prolongation in GI-residence time of nanocarriers will improve efficiency in transversing intestinal epithelium, thereby improving bioavailability ${ }^{26,39,58}$. This ought to get improved by decreasing their size below $200 \mathrm{~nm}$, which in turn will diffuse through mucus and circumvent elimination by mucilliary clearance. Mucoadhesion translates to cumulative protein release and absorption ${ }^{39,59}$, 64

\section{DISCUSSION AND STRATEGY FOR DEVELOPMENT}

Nowadays NBODSM considered as promising alternative to parenteral one. $\mathrm{pH}$ sensitive, untargeted, or targeted (with or without grafting of ligand) NBODSM had been exploited in most cases 1, 5, 16, 30, 65. Future of their marketing remains uncertain due to following facts.

(i) High cost of synthesising polymer, manufacturing nanocarriers and scaling-up,

(ii) Improvement of bioavailability to stable and efficient therapeutic level,

(iii) High inter- and intra-individual variations in pharmacokinetics of macromolecules, and

(iv) Optimizing polymeric composition of them for specific macromolecules.

Selection of nanocarrier remains controversial for delivering macromolecules. Amenability of NPs for modulation in physicochemical and biological behaviour makes them versatile, thus suitable for delivering wide variety of drug with or without target specificity ${ }^{5}$. Micelles considered viable approach for delivering poorly soluble macromolecules comparing cyclodextrins based solid dispersions or lipid-based systems ${ }^{13}, 19,36,38 \cdot \mathrm{pH}^{- \text {-sensitive }}$ micelles minimises initial burst release of macromolecules

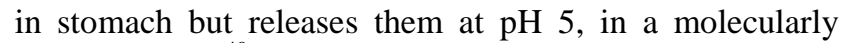
dispersed form ${ }^{40}$. LIP and lipospheres have lower toxicity and higher tolerability, and suitable for poorly soluble macromolecules ${ }^{45,}{ }^{46}$.SLN, comprises physiological and well-tolerated lipids, offer controlled release and targeting, provide protection against degradation, and is advantageous over other colloidal carriers $12,42,44,66,67$.

Size of nanocarriers should be essentially within 10-200 $\mathrm{nm}$, to promote diffusion in mucus and uptake by intestinal cells, and to decrease uptake by reticulo-endothelial system (RES) ${ }^{16}$. Surface modification of them with PEG will prevent their uptake by RES and prolong circulation 
half-life ${ }^{21,} 25$. Imparting mucoadhesion property will prolong their residence time without impeding diffusion in mucus $39,58,59$. Their surface charge has to be positive favouring interaction with mucus and cell membrane but be neutral to decrease RES clearance ${ }^{26,39,64}$.

The mechanisms of drug absorption from micelles differ from NPs uptake. Nanocarriers should be stable in GI tract while releasing drug at desired site with predetermined rate. They may have targeting potentiality for $\mathrm{M}$ cells or immunocompetent cells of GI tract ${ }^{9,38,47,50}$.

Chitosan have ability to cause reversible opening of TJs and have mucoadhesive property ${ }^{29,32,33,68}$.Improvement in paracellular permeability did not increases permeability of endotoxins and lipopolysaccharide thus is safe ${ }^{69}$. Polyelectrolyte complexation of drug with chitosan prevents enzymatic degradation in GI environment ${ }^{28}$. Hydrogel system of alginate, dextran sulphate and poloxamer 188 consisting multilayered NPs of chitosan improves entrapment and gastric retention. Poloxamers improves solubility and transport of drug 8, 41, 48 . Decreasing concentration of $\mathrm{Ca}^{2+}$ and increasing concentration of poloxamer and albumin results smaller NPs. Reduction in albumin content improves entrapment and gastric retention ${ }^{10,11}$.

Loss in mucoadhesive and TJopening properties of chitosan at pH 7.4 is improved by derivatisation ${ }^{29,33,34}$. Ntrimethylated chitosan (NTC), thiolated chitosan (TC) ${ }^{68}$, N-(2-hydroxyl) propyl-3- trimethyl ammonium chitosan $\left(\mathrm{NTAC}^{70}\right.$, are important derivatives ${ }^{29}$. These categorised as $\mathrm{pH}$-sensitive polymers used for improving bioavailability alone or in combination with other polymer 4, 71. PEGylatedchitosan have enhanced permeability and lower toxicity ${ }^{25}$. Gama-polyglutamic acid ( $\gamma$ PGA)-NTC based NPs allow absorption of macromolecules throughout the intestinal tract ${ }^{71,72}$.

NTC based NPs involve active transport and electrostatic interaction, which upon conjugation with ligand CKS9, CSC enhances absorption, further, via clathrin- and caveolae- mediated endocytosis 23, 53, 56, 57.From $\mathrm{pH}$ responsive NPs macromolecules gets absorbed into systemic circulation while carrier retained mainly in oral tract 65,73 . Some $\mathrm{pH}$-responsive nanocarriers are biodegradable ${ }^{40}$.TC improves mucoadhesion and absorption eliminating toxicity and biocompatibility issue ${ }^{68}$. NTAC exhibit higher cationic charge density, increased mucoadhesion, TJ opening ability and were non-cytotoxic 70

NPs of chitosan- $\gamma$ PGA increase their absorption in intestine and sustain release. Their conjugation with CSC results targeting of goblet cells, but with diethylenetriaminepentaaceticacid disruptsTJs ${ }^{71}$. Blending chitosan and $\gamma \mathrm{PGA}$ with tripolyphosphate and $\mathrm{MgSO}_{4}$ result multi-ion-crosslinked NPs, possessing stability over broader $\mathrm{pH}$ ranges and improved transporting capability, and more compact one ${ }^{8,29}$.

Acrylic-based polymers and copolymers like poly(isobutylcyanoacrylate) (PIBCA), poly(acrylic acid) (PAA), poly(alkylcyanoacrylate), PEG grafted methacrylic acid (MAA) or acrylic acid used to get $\mathrm{pH}$ sensitive NPs. Copolymerising MAA with PEG dimethacrylate (PG(c) 2011, JDDT. All Rights Reserved
DMAA) having molecular weights of $400-4000 \mathrm{~g} / \mathrm{mol}$ results hydrophilic and $\mathrm{pH}$-sensitive poly(PG-DMAAMAA) NPs $5,7,25,30$.Modification of dextran based NPs through conjugation of its surface with vitamin $B_{12}$ results targeting with better transport and higher bioavailability ${ }^{60}$.Thiolatedpoly(acrylic acid) or it's blend with chitosan improves mucoadhesive properties of NPs ${ }^{68}$, 74

PLGA NPs gets distributed in liver, spleen, lungs, brain and kidneys, while majority were located in liver but these upon PEG-grafting rapidly taken up by peritoneal macrophages ${ }^{31}$. PLGA or its blend with $\beta$-cyclodextrin (PLGA- $\beta C D)$, hydroxypropyl methylcellulose phthalate (PLGA-HP55) and Eudragit@ RS (PLGA-ERS) are also exploited ${ }^{75}$. PLGA- $\beta C D$, PLGA-HP55 and star-branched form of PLGA exhibit burst release effect. While PLGAERS based NPs remains attached to intestinal epithelium and able to open $\mathrm{TJs}^{75} \cdot \gamma$ PGAgraftedmesoporoussilicaNPs are $\mathrm{pH}$-sensitive and possess much higher release rate at pH 5.5 comparing $\mathrm{pH} 7.4{ }^{72}$.PLGA based NPs upon coating with chitosan exhibit improved mucoadhesion ${ }^{59}$. Co-encapsulating antacids (viz. magnesium hydroxide, magnesium carbonate or zinc carbonate) in PLGA NPs preserve the structure of macromolecules and protect them from GI tract degradation ${ }^{76}$. PLGA-based NPs targeting M cells might be more efficient for vaccine delivery that sustain release and deliver antigen directly to the immune cells and might achieve and have intrinsic adjuvanteffect on DCs $31,57,77$.PCL-ERS, and poly(allylamine) are alternatives for getting mucoadhesive NPs.

Dipalmitoyl-phosphatidylcholine (DPPC), egg, phosphatidylcholine, or phosphatidylinositol-cholesterol based LIP are used while DPPC offers protection of drug against degradation in GI tract and their entry in bloodstream, intact ${ }^{45,46}$. Surface conjugation or coatings of LIP with mucin, PEG, wheat germ agglutinin (WGA), tomato lectin (TL) and Ulexeuropaeus agglutinin 1 (UA1) results targeting. Mucin-LIP and PEG-LIP results sustained release and provides protection against bile salts $1,45,46$. LIP-WGA is taken up by endocytosis, LIP-TL exhibits resistance to enzymatic action in the intestine and mucoadhesion, LIP-UA1 have targeting specificity to M cells of PPs 9, 45, 56, 57. Co-encapsulation of permeation enhancers (e.g. sodium taurocholate, dimethyl palmitoylammoniopropanesulfonate) enhances bioavailability and paracellular translocation, through disrupting $\mathrm{TJ}^{78}$.

Poly(allylamine) are amphiphilic and protects macromolecules from degradation by pepsin, trypsin and chymotrypsin along with improvement of transcellular and paracellular transport while latter being via reversible disruption of $\mathrm{TJ}_{\mathrm{S}}{ }^{28,79}$. PLA-Pluronic block copolymer based vesicles have ability to pass through cell membranes and attracted to small intestine ${ }^{63}$. Copolymer of pluronicPAA and poloxamer minimise initial burst release of drug, in the acidic stomach, but releases drug, in a molecularly dispersed form, at $\mathrm{pH}$ five. Targeting and mucoadhesion property of these synergises efficiency and efficacy ${ }^{39}$. SLN ofcetylpalmitate sustain release while that of WGA$\mathrm{N}$-glutarylphosphatidylethanolamine exhibits protection against enzymatic degradation ${ }^{11,12,43,67,80}$.Micelles of 
monomethyl

PEG750-poly(caprolactone-cotrimethylcarbonate), copolymer, confers 1 to 3 fold increase in solubility of poorly soluble drugs and bioavailability ${ }^{14,18}$.

To avert drug release in GI tract, concentration of micelle to be above critical micelle concentration (CMC) and it shall expose to an ionic strength below their flocculation point ${ }^{27,81}$. The inhibition of P-gp by pluronics is highest just below CMCwhereas that of PCL-PEG is above CMC 14,18 . Micelles of poorly soluble drugs could be passively target by enhanced permeabilisation-retention effect ${ }^{14}$. Poly-ion complex micelle enhances transport across intestinal epithelial barrier ${ }^{27,28}$.

Pluronics, poloxamers, TPGS and PCL-PEG inhibit P-gp and enhance net drug transport through intestinal barrier ${ }^{14}$, 18, 41, ${ }^{63}$.Some NPs enhances transcytosis through proteaseinhibition $^{82}$. Multilayered NPs of alginate colocalises in the small intestinal mucosa. Amine-modified graft polyesters-chitosan NPs or thiolatedtrimethylchitosan NPs conserving degradation of macromolecules and facilitate their uptake, while latter had intrinsic adjuvanteffect on DCs ${ }^{48,77}$.

Lipid-based systems (lipospheres, micelles, and LIP), prepared with several lipids including orexcluding emulsifying agents, well tolerated in living systems did not exhibit any cytotoxic effects, up to total of $2.5 \%$ lipid content. LIPisadvantageous in terms of amphiphiliccharacter, biocompatibility, and ease of surface modification.SLNis innovative system having expectationfor more contributions, particularly,poor aqueous soluble macromolecules. Considering tolerability, surfactant of GRAS status used with preference, comparing lipids. Acylglycerols composed of fatty acids and lecithins mostly accepted as safe ${ }^{19,38}$.

Surface charges or chemistry of nanocarriers seem to monitor their fate. Negatively charged one display higher transport rates referring near neutral, or positively charged $^{5,64}$. Fast-diffusing nanocarriers are achievable by coating their surface with PEG. This surface engineering modulates their mucoadhesive properties. Dense coating effectively minimises adhesive interactions of them with mucins, allowing penetration ${ }^{21}$.GI-residence time can be improved with cationic polymers or coating them with cationic groups or with thiol groups (binds to mucin) ${ }^{21,35}$. However, a balance between mucoadhesion and mucus penetration is important ${ }^{5}$.

Nanocarriers must be small enough to avoid significant steric inhibition by the fibre mesh and should avoid adhesion to mucin fibres; and to allow diffusion and uptake, but large enough to carry a favourable amount of macromolecules ${ }^{48}$. They being stable, non-toxic, bioabsorbable, non-thrombogenic, non-immunogenic, non-

\section{REFERENCES}

1. Saikh MAA. Nanoscience and nanotechnology: Issues and Applications in agri-food, biomedical, and environmental sciences. International Journal of Pharmaceutical Frontier Research, 2012, 2(1), 61-86.

2. Petkar KC, Chavhan SS, Agatonovik-Kustrin S, Sawant KK, Nanostructured materials in drug and gene delivery: A review of inflammatory, avoid uptake by RES and have versatile applicability $1,17,20$. Cytotoxicity of nanovesicles is an essential product parameter of in vivo tolerance evaluation in humans and/or animals ${ }^{17,19,38}$. Their stability assessed involving approved method and accordingly shelf-life be assigned $^{39}$.

Targeting of nanocarriers to globet cell ${ }^{53,54} \mathrm{PPs}{ }^{9,45,56} \mathrm{DCs}$ of PPs ${ }^{57,62}$ achieved with grafting of ligands. Optimisation of ligand density on their surface must allow tissue penetration and cellular uptake resulting in optimal therapeutic efficacy. Redressing issue of variation in $\mathrm{M}$ cells populations and receptors needed; those vary with species, anatomical location, age, sex and exogenous factors $1,9,10$

In vitro models used for understanding fate of nanocarriers within intestinal epithelium is critical for their development. Combining both quantitative analysis method studying their transport and confocal microscopy, locating them, improve efficiency of study ${ }^{39,83}$. Cellular tracking of them can be done with quantum dots technique that improves their visualization and dynamic colocalization microscopy 61,83 . Quantitative and qualitative information such as diffusivity, viscoelasticity, pore size, velocity, directionality and transport mode of drug vectors can be determined from particle trajectories that reflect transport in mucus. 3-(4,5-dimethylthiazol-2yl)-2,5-diphenyltetrazolium bromide assays provide cytotoxic report of the nanocarriers ${ }^{70}$. Association of nanocarriers with DCs and their effect on their maturation determined flow-cytometrically ${ }^{77}$.

Novel NBODSM can be developed through ${ }^{1}$.

(i) Development of biocompatible polymers with tailored attributes for orally administered nanocarriers,

(ii) Understanding the mechanisms of their cellular uptake,

(iii) Devising novel techniques for studying their fate

including its components, and

(iv) Identifying ligands for their targeted delivery.

\section{CONCLUSION}

NBODSM will be a promising platform for orally delivering macromolecules. In vivo experimental results of animal models need to be interpreted and correlated with care, associated with existence of differences in anatomy and physiology of laboratory animals and humans. In order have successful NBODSM bioavailability of them to reach therapeutic level, with minimal inter and intra individual variation and toxicity had to be considered. Greater stability along with a financially feasible manufacturing process for mass production is prerequisite. Future will evidence utility of NBODSM in devising projected benefit in a cost-effective and sustainable way. Step to set up to arouse professional in delivering worthwhile effects to society. the state of the art, Critical Reviews in Therapeutic Drug Carrier Systems, 2011, 28(2), 101-164.

3. Moutinho CG, Matos CM, Teixeira JA, Balcão VM, Nanocarrier possibilities for functional targeting of bioactive peptides and proteins: State-of-the-art, Journal of Drug Targeting, 2012, 20(2), 114-141. 
4. Sonaje K, Lin KJ, Wang JJ, Mi FL, Chen CT, Juang JH, Sung HW,Self-assembled ph-sensitive nanoparticles: A Platform for oral delivery of protein drugs, Advanced Functional Materials, 2010, 20(21), 3695-3700.

5. Hunter AC, Elsom J, Wibroe PP, Moghimi SM, Polymeric particulate technologies for oral drug delivery and targeting: A Pathophysiological perspective, Maturitas, 2012, 73(1), 5-18.

6. Ruenraroengsak P, Cook JM, Florence AT, Nanosystemdrugtargeting: Facingup to complexrealities, Journal of Controlled Release, 2010, 141(3), 265-276.

7. Herrero EP, Alonso MJ, Csaba N, Polymer-based oral peptide nanomedicines, Therapeutic Delivery, 2012, 3(5), 657-668.

8. Roger E, Lagarce F, Garcion E, Benoit JP, Biopharmaceutical parameters to consider in order to alter the fate of nanocarriers after oral delivery,Nanomedicine (London), 2010, 5(2), 287-306.

9. Brayden DJ, Jepson MA, Baird AW, Keynote review: Intestinal Peyer's patch $\mathrm{M}$ cells and oral vaccine targeting, Drug Discovery Today, 2005, 10(17), 1145-1157.

10. Alexis F, Pridgen E, Molnar LK, Farokhzad OC, Factors affecting the clearance and biodistribution of polymeric nanoparticles, Molecular Pharmacology, 2008, 5(4), 505-515.

11. Qi J, Lu Y, Wu W, Absorption, disposition and pharmacokinetics of solidlipidnanoparticles, Current Drug Metabolism, 2012, 13(4), 418-428.

12. Bondì ML, Craparo EF, Solidlipidnanoparticles for applications in gene therapy: A review of the state of the art, Expert Opinion on Drug Delivery, 2010, 7(1), 7-18.

13. Iqbal MA, Md S, Sahni JK, Baboota S, Dang S, Ali J, Nanostructured lipid carriers system: Recent Advances in drug delivery, Journal of Drug Targeting, 2012, 20(10), 813-830.

14. Chen D, Ding PT, Deng YH, Wang SL, Advances in the study of polymeric micelles used in oral administration, Yao XueXueBao, 2010, 45(5), 560-564.

15. $\mathrm{Xu} \mathrm{W}$, Ling $\mathrm{P}$, Zhang $\mathrm{T}$, Polymericmicelles, a promisingdrug delivery system to enhancebioavailability of poorlywatersolubledrugs, Journal of Drug Delivery, 2013, 2013, 340315

16. Rabanel JM, Aoun V, Elkin I, Mokhtar M, Hildgen P, Drugloaded nanocarriers: Passive targeting and crossing of biological barriers, Current Medicinal Chemistry, 2012, 19(19), 3070-3102.

17. Jian F, Zhang Y, Wang J, Ba K, Mao R, Lai W, Lin Y, Toxicity of biodegradable nanoscale preparations, Current Drug Metabolism, 2012, 13(4), 440-446.

18. Gaucher G, Satturwar P, Jones MC, Furtos A, Leroux JC, Polymeric micelles for oral drug delivery, European Journal of Pharmaceutics and Biopharmaceutics, 2010, 76(2), 147-158.

19. Li P, Nielsen HM, Müllertz A, Oral delivery of peptides and proteins using lipid-based drug delivery systems,Expert Opinion on Drug Delivery, 2012, 9(10), 1289-1304.

20. Kumari A, Yadav SK, Yadav SC, Biodegradable polymeric nanoparticles based drug delivery systems, Colloids and Surfaces B: Biointerfaces, 2010, 75(1), 1-18.

21. Wang YY, Lai SK, Suk JS, Pace A, Cone R, Hanes J, Addressing the PEGmucoadhesivityparadox to engineernanoparticles that "slip" through the humanmucusbarrier, AngewandteChemie International Edition (English), 2008, 47(50), 9726-9729.

22. Deli MA, Potential use of tight junctionmodulators to reversiblyopenmembranousbarriers and improvedrug delivery, Biochimica et BiophysicaActa, 2009, 1788(4), 892-910.

23. Muro S, Challenges in design and characterization of ligandtargeteddrug delivery systems, Journal of Controlled Release, 2012, 164(2), 125-137.

24. Fievez V, Plapied L, Plaideau C, Legendre D, des Rieux A, Pourcelle V, Freichels H, Jérôme C, Marchand J, Préat V, Schneider YJ, In vitro identification of targeting ligands of human $\mathrm{M}$ cells by phage display, International Journal of Pharmaceutics, 2010, 394(1-2), 35-42.

25. Casettari L, Vllasaliu D, Mantovani G, Howdle SM, Stolnik S, Illum L, Effect of PEGylation on the toxicity and permeability enhancement of chitosan, Biomacromolecules, 2010, 11(11), 2854-2865.

26. Ensign LM, Cone R, Hanes J, Oral drug delivery with polymeric nanoparticles: The Gastrointestinal mucus barriers, Advanced Drug Delivery Reviews, 2012, 64(6), 557-570.
27. Li N, Li XR, Zhou YX, Li WJ, Zhao Y, Ma SJ, Li JW, Gao YJ, Liu Y, Wang XL, Yin DD, The use of polyion complex micelles to enhance the oral delivery of salmon calcitonin and transport mechanism across the intestinal epithelial barrier, Biomaterials, 2012, 33(34), 8881-8892.

28. Thompson C, Cheng WP, Gadad P, Skene K, Smith M, Smith G, McKinnon A, Knott R, Uptake and transport of novelamphiphilicpolyelectrolyte-insulinnanocomplexes by Caco2cells--towards oral insulin, Pharmaceutical Research, 2011, 28(4), 886-896.

29. Chaudhury A, Das S, Recentadvancement of chitosanbasednanoparticles for oralcontrolleddelivery of insulin and othertherapeuticagents, AAPS PharmSciTech, 2011, 12(1), 1020.

30. Duncan R, Vicent MJ, Polymer therapeutics-prospects for 21st century: The End of the beginning, Advanced Drug Delivery Reviews, 2013, 65(1), 60-70.

31. Semete B, Booysen LI, Kalombo L, Venter JD, Katata L, Ramalapa B, Verschoor JA, Swai H, In vivouptake and acuteimmuneresponse to orallyadministeredchitosan and PEGcoatedPLGAnanoparticles, Toxicology and Applied Pharmacology, 2010, 249(2), 158-165.

32. Hsu LW, Lee PL, Chen CT, Mi FL, Juang JH, Hwang SM, Ho YC, Sung HW, Elucidating the signaling mechanism of an epithelialtight-junction opening induced by chitosan, Biomaterials, 2012, 33(26), 6254-6263.

33. Yeh TH, Hsu LW, Tseng MT, Lee PL, Sonjae K, Ho YC, Sung HW, Mechanism and consequence of chitosan-mediated reversible epithelialtight junction opening, Biomaterials, 2011, 32(26), 6164-6173.

34. Werle M, Takeuchi H, Bernkop-Schnürch A, Modifiedchitosans for oraldrug delivery,Journal of Pharmaceutical Sciences, 2009, 98(5), 1643-1656

35. Tang BC, Dawson M, Lai SK, Wang YY, Suk JS, Yang M, Zeitlin P, Boyle MP, Fu J, Hanes J, Biodegradablepolymernanoparticles that rapidlypenetrate the humanmucusbarrier, Proceedings of the National Academy of Sciences of the United States of America, 2009, 106(46), 1926819273.

36. Musacchio T, Torchilin VP, Recent developments in lipid-based pharmaceutical nanocarriers, Frontiers in Bioscience (Landmark Edition), 2011, 16, 1388-1412.

37. Das S, Chaudhury A, Recent advances in lipid nanoparticle formulations with solid matrix for oral drug delivery, AAPS PharmSciTech, 2011, 12(1), 62-76.

38. Martins S, Sarmento B, Ferreira DC, Souto EB, Lipid-based colloidal carriers for peptide and protein delivery--liposomes versus lipidnanoparticles, International Journal of Nanomedicine, 2007, 2(4), 595-607.

39. Saikh MAA. Gastro retentive drug delivery system: An Update. World Journal of Pharmaceutical Research, 2013, 2(3): 297-307.

40. Chen J, Ouyang J, Kong J, Zhong W, Xing MM, Photo-crosslinked and $\mathrm{pH}$-sensitive biodegradable micelles for doxorubicin delivery, ACS Applied Materials \& Interfaces, 2013, 5(8), 31083117.

41. Sahay G, Batrakova EV, Kabanov AV, Different internalization pathways of polymericmicelles and unimers and their effects on vesicular transport, Bioconjugate Chemistry, 2008, 19(10), 20232029.

42. Battaglia L, Gallarate M, Lipidnanoparticles: State of the art, new preparation methods and challenges in drug delivery, Expert Opinion on Drug Delivery, 2012, 9(5), 497-508.

43. Parhi R, Suresh P, Preparation and characterization of solidlipidnanoparticles-A Review, Current Drug Discovery Technologies, 2012, 9(1), 2-16.

44. Corrias F, Lai F, New methods for lipidnanoparticles preparation, Recent Patents on Drug Delivery \& Formulation, 2011, 5(3), 201-213.

45. Elgart A, Cherniakov I, Aldouby Y, Domb AJ, Hoffman A, Lipospheres and pro-nanolipospheres for delivery of poorly water soluble compounds, Chemistry and Physics of Lipids, 2012, 165(4), 438-453. 
46. Swaminathan J, Ehrhardt C, Liposomal delivery of proteins and peptides, Expert Opinion on Drug Delivery, 2012, 9(12), 14891503.

47. Duncan R, Richardson SC, Endocytosis and intracellular trafficking as gateways for nanomedicine delivery: Opportunities and challenges, Molecular Pharmacology, 2012, 9(9), 2380-2402.

48. Plapied L, Duhem N, des Rieux A, Préat V, Fate of polymeric nanocarriers for oral drug delivery, Current Opinion in Colloid \& Interface Science, 2011, 16(3), 228-237.

49. Oostendorp RL, Beijnen JH, Schellens JH, The biological and clinicalrole of drugtransporters at the intestinalbarrier, Cancer Treatment Reviews, 2009, 35(2), 137-147.

50. Zaki NM, Tirelli N, Gateways for the intracellular access of nanocarriers: A Review of receptor-mediated endocytosis mechanisms and of strategies in receptor targeting, Expert Opinion on Drug Delivery, 2010, 7(8), 895-913.

51. Bareford LM, Swaan PW, Endocyticmechanisms for targeteddrug delivery, Advanced Drug Delivery Reviews, 2007, 59(8), 748-758.

52. Cai Z, Wang Y, Zhu LJ, Liu ZQ, Nanocarriers: A General strategy for enhancement of oral bioavailability of poorly absorbed or pre-systemically metabolized drugs, Current Drug Metabolism, 2010, 11(2), 197-207.

53. Jin Y, Song Y, Zhu X, Zhou D, Chen C, Zhang Z, Huang Y, Gobletcell-targetingnanoparticles for oralinsulindelivery and the influence of mucus on insulintransport,Biomaterials, 2012, 33(5), 1573-1582.

54. Marelli UK, Rechenmacher F, Sobahi TR, Mas-Moruno C, Kessler H,Tumor targeting via integrin ligands,Frontiers in Oncology, 2013, 3, 222.

55. Kyd JM, Cripps AW, Functionaldifferences between Mcells and enterocytes in samplingluminalantigens, Vaccine, 2008, 26(49), 6221-6224.

56. Yoo MK, Kang SK, Choi JH, Park IK, Na HS, Lee HC, Kim EB, Lee NK, Nah JW, Choi YJ, Cho CS, Targeteddelivery of chitosannanoparticles to Peyer'spatch using Mcellhomingpeptideselected by phagedisplaytechnique, Biomaterials, 2010, 31(30), 7738-7747.

57. Fievez V, Plapied L, des Rieux A, Pourcelle V, Freichels H, Wascotte V, Vanderhaeghen ML, Jerôme C, Vanderplasschen A, Marchand-Brynaert J, Schneider YJ, Préat V, Targeting nanoparticles to $\mathrm{M}$ cells with non-peptidic ligands for oral vaccination, European Journal of Pharmaceutics and Biopharmaceutics, 2009, 73(1), 16-24.

58. Salman HH, Gamazo $\mathrm{C}$, Agüeros $\mathrm{M}$, Irache JM, Bioadhesivecapacity and immunoadjuvantproperties of thiaminecoatednanoparticles, Vaccine, 2007, 25(48), 8123-8132.

59. Zhang X, Sun M, Zheng A, Cao D, Bi Y, Sun J, Preparation and characterization of insulin-loadedbioadhesivePLGAnanoparticles for oraladministration, European Journal of Pharmaceutical Sciences, 2012, 45(5), 632-638.

60. Petrus AK, Fairchild TJ, Doyle RP, Traveling the vitaminB12pathway: OralDelivery of protein and peptidedrugs, AngewandteChemie International Edition (English), 2009, 48(6), 1022-1028.

61. Gao X, Wang T, Wu B, Chen J, Chen J, Yue Y, Dai N, Chen H, Jiang X, Quantum dots for trackingcellulartransport of lectinfunctionalizednanoparticles, Biochemical and Biophysical Research Communications, 2008, 377(1), 35-40.

62. Primard C, Rochereau N, Luciani E, Genin C, Delair T, Paul S, Verrier B, Traffic of poly(lactic acid) nanoparticulate vaccine vehicle from intestinal mucus to sub-epithelial immune competent cells, Biomaterials, 2010, 31(23), 6060-6068.

63. Batrakova EV, Kabanov AV,Pluronicblockcopolymers: Evolution of drug deliveryconcept from inertnanocarriers to biological response modifiers, Journal of Controlled Release, 2008, 130(2), 98-106.

64. Crater JS, Carrier RL, Barrierproperties of gastrointestinalmucus to nanoparticletransport, Macromolecular Bioscience, 2010, 10(12), 1473-1483.

65. Wang Y, Chang B, Yang W, pH-sensitivepolyketalnanoparticles for drug delivery, Journal of Nanoscience and Nanotechnology, 2012, 12(11), 8266-8275.
66. Bunjes H, Lipidnanoparticles for the delivery of poorly watersoluble drugs, Journal of Pharmacy and Pharmacology, 2010, 62(11), 1637-1645.

67. Harde H, Das M, Jain S, Solidlipidnanoparticles: An Ora bioavailability enhancer vehicle,Expert Opinion on Drug Delivery, 2011, 8(11), 1407-1424.

68. Yin L, Ding J, He C, Cui L, Tang C, Yin C, Drugpermeability and mucoadhesionproperties of thiolatedtrimethylchitosan nanoparticles in oral insulin delivery, Biomaterials, 2009, 30(29), 5691-5700.

69. Sonaje K, Lin KJ, Tseng MT, Wey SP, Su FY, Chuang EY, Hsu CW, Chen CT, Sung HW, Effects of chitosan-nanoparticlemediatedtightjunctionopening on the oralabsorption of endotoxins, Biomaterials, 2011, 32(33), 8712-8721.

70. Sonia TA, Sharma CP, In vitro evaluation of N-(2-hydroxy) propyl-3-trimethyl ammonium chitosan for oral insulin delivery, Carbohydrate Polymers, 2011, 84(1),103-109.

71. Sonaje K, Chen YJ, Chen HL, Wey SP, Juang JH, Nguyen HN, Hsu CW, Lin KJ, Sung HW, Enteric-coatedcapsulesfilled with freeze-driedchitosan/poly(gamma-glutamicacid) nanoparticles for oralinsulindelivery, Biomaterials, 2010, 31(12), 3384-3394.

72. Zheng J, Tian X, Sun Y, Lu D, Yang W, pH-sensitive poly(glutamic acid) grafted mesoporous silica nanoparticles for drug delivery, International Journal of Pharmaceutics, 2013, 450(1-2), 296-303.

73. Wang XQ, Zhang Q, pH-sensitive polymeric nanoparticles to improve oral bioavailability of peptide/protein drugs and poorly water-soluble drugs, European Journal of Pharmaceutics and Biopharmaceutics, 2012, 82(2), 219-229.

74. $\mathrm{Xu} \mathrm{J}$, Gattacceca F, Amiji M, Biodistribution and pharmacokinetics of EGFR-targeted thiolatedgelatinnanoparticles following systemic administration in pancreatic tumor-bearing mice, Molecular Pharmacology, 2013, 10(5), 2031-2044.

75. Wu ZM, Zhou L, Guo XD, Jiang W, Ling L, Qian Y, Luo KQ, Zhang LJ, HP55-coatedcapsulecontainingPLGA/RSnanoparticles for oraldelivery of insulin, International Journal of Pharmaceutics, 2012, 425(1-2), 1-8.

76. Sharma G, van der Walle CF, Ravi Kumar MN, Antacidcoencapsulatedpolyesternanoparticles for peroraldelivery of insulin: Development, pharmacokinetics, biodistribution and pharmacodynamics, International Journal of Pharmaceutics, 2013, 440(1), 99-110.

77. Slütter B, Plapied L, Fievez V, Sande MA, des Rieux A, Schneider YJ, Van Riet E, Jiskoot W, Préat V, Mechanisticstudy of the adjuvanteffect of biodegradablenanoparticles in mucosalvaccination, Journal of Controlled Release, 2009, 138(2), 113-121.

78. Gupta V, Hwang BH, Doshi N, Mitragotri S, A permeation enhancer for increasing transport of therapeutic macromolecules across the intestine, Journal of Controlled Release, 2013, [In press]

79. Thompson CJ, Tetley L, Cheng WP, The influence of polymerarchitecture on the protectiveeffect of novelcombshapedamphiphilicpoly(allylamine) against in vitroenzymaticdegradation of insulin-towardsoralinsulindelivery, International Journal of Pharmaceutics, 2010, 383(1-2), 216-227.

80. Pathak K, Keshri L, Shah M,Lipidnanocarriers: Influence of lipids on product development and pharmacokinetics,Critical Reviews in Therapeutic Drug Carrier Systems, 2011, 28(4), 357393.

81. Li SD, Huang L,Pharmacokinetics and biodistribution of nanoparticles,Molecular Pharmacology, 2008, 5(4), 496-504

82. Su FY, Lin KJ, Sonaje K, Wey SP, Yen TC, Ho YC, Panda N, Chuang EY, Maiti B, Sung HW, Proteaseinhibition and absorptionenhancement by functionalnanoparticles for effectiveoralinsulindelivery, Biomaterials, 2012, 33(9), 28012811.

83. Vercauteren D, Deschout H, Remaut K, Engbersen JF, Jones AT, Demeester J, De Smedt SC, Braeckmans K, Dynamic colocalization microscopy to characterize intracellular trafficking of nanomedicines, ACS Nano, 2011, 5(10), 7874-7884. 